PROCEEDINGS OF THE

AMERICAN MATHEMATICAL SOCIETY

Volume 130, Number 10, Pages 2827-2836

S 0002-9939(02)06421-3

Article electronically published on March 12, 2002

\title{
ON LOCALLY FINITE $p$-GROUPS SATISFYING AN ENGEL CONDITION
}

\author{
ALIREZA ABDOLLAHI AND GUNNAR TRAUSTASON
}

(Communicated by Stephen D. Smith)

\begin{abstract}
For a given positive integer $n$ and a given prime number $p$, let $r=r(n, p)$ be the integer satisfying $p^{r-1}<n \leq p^{r}$. We show that every locally finite $p$-group, satisfying the $n$-Engel identity, is (nilpotent of $n$-bounded class)by-(finite exponent) where the best upper bound for the exponent is either $p^{r}$ or $p^{r-1}$ if $p$ is odd. When $p=2$ the best upper bound is $p^{r-1}, p^{r}$ or $p^{r+1}$. In the second part of the paper we focus our attention on 4-Engel groups. With the aid of the results of the first part we show that every 4-Engel 3-group is soluble and the derived length is bounded by some constant.
\end{abstract}

\section{INTRODUCTION}

A group is said to be an $n$-Engel group if it satisfies the law $\left[x,_{n} y\right]=1$, where $\left[x,_{m} y\right]$ is defined inductively by $\left[x,_{0} y\right]=x$ and $\left[x,_{m+1} y\right]=\left[\left[x,_{m} y\right], y\right]=$ $\left[x,_{m} y\right]^{-1} y^{-1}\left[x,_{m} y\right] y$. In the first part of this paper we investigate locally finite $p$-groups that satisfy an $n$-Engel identity. Our results in this first part rely on the following two deep theorems of E. Zel'manov [26], [28], [29].

Theorem Z1. Every n-Engel Lie algebra over a field of characteristic zero is nilpotent.

Theorem Z2. Every n-Engel Lie algebra is locally nilpotent.

In fact we will apply the following corollaries of Zel'manov's results. The first is due to Zel'manov [27] and the second to J. Wilson [25]. For a short proof of the latter see [4].

Theorem Z3. Every torsion free locally nilpotent n-Engel group is nilpotent of $n$-bounded class.

Theorem W. Every residually finite $n$-Engel group is locally nilpotent.

Let $n$ be a given positive integer and $p$ a given prime number. Let $r=r(n, p)$ be the integer satisfying $p^{r-1}<n \leq p^{r}$. If $p$ is odd, we will show that all locally finite $n$-Engel $p$-groups are (nilpotent of $n$-bounded class)-by-(finite exponent), where the exponent divides $p^{r}$. We will also show that either $p^{r}$ or $p^{r-1}$ is the best upper

Received by the editors March 26, 2001 and, in revised form, May 12, 2001.

2000 Mathematics Subject Classification. Primary 20F45, 20F50.

Key words and phrases. Locally finite $p$-groups, Engel groups.

The second author thanks the European Community for their support with a Marie Curie grant. 
bound. For $p=2$ we will show that $\left(G^{2^{r}}\right)^{2}$ is nilpotent. So here again $G$ is (nilpotent of $n$-bounded class)-by-(finite exponent) but the best upper bound is either $2^{r-1}, 2^{r}$ or $2^{r+1}$. We remark here that in [5], the authors already show that locally finite $n$-Engel $p$-groups are nilpotent (by finite exponent) but the proof is different and they do not obtain our sharp upper bound for the exponent.

Our proof also uses some theory of powerful $p$-groups. The reader can find most of the material that we use in [6] or [17].

In the second part of the paper we will work on 4-Engel groups. Whereas 3Engel groups are now quite well understood (see for example [2], [10, 12, [13, [15]), relatively little is known about the structure of 4-Engel groups. In particular it is still unknown whether every 4-Engel $p$-group needs to be locally finite. In 22 it was shown that this is true if $p=2$ or $p=3$ and for other primes $p$, the problem was reduced to determining whether 4-Engel groups of exponent $p$ are locally finite. In 24 M. Vaughan-Lee proved that 4-Engel groups of exponent 5 are locally finite but for primes greater than 5 the question remains open. However, if $p \geq 7$, then every locally finite 4 -Engel $p$-group is nilpotent of class at most 7 [22] (see also [9], 21]).

There are 4-Engel $p$-groups that are not nilpotent if $p=2,3$ or 5 . This is easily seen for $p=2$ or 3 by considering a standard wreath product of a cyclic group of order $p$ by an elementary abelian $p$-group of countable rank. Then in [2], Bachmuth and Mochizuki constructed an example of a 3-Engel group of exponent 5 that is not soluble. By looking at a power commutator presentation of the free 3 -generator group of exponent 4 [3], one sees that every group of exponent 4 is central by 4-Engel. As groups of exponent 4 need not be soluble [18], the same is true for 4-Engel groups of exponent 4. We should add here that all 3-Engel 2-groups are on the other hand soluble [10].

There remains the question of whether 4-Engel 3-groups are soluble. With the aid of the results of the first part, we will show that 4-Engel 3-groups are soluble and that the derived length is bounded by some constant. We will see that it follows from this that any locally nilpotent 4-Engel group without elements of order 2 or 5 is soluble. This answers question 9.50.(a) in the Kourovka notebook 23] for locally nilpotent groups. This was also conjectured by S. Bachmuth [1.

\section{LOCALLY FINITE $p$-GROUPS}

In this section, we let $n$ be a fixed positive integer, $p$ be a fixed prime number and $r=r(n, p)$ be the integer satisfying $p^{r-1}<n \leq p^{r}$. Our results will depend on the following corollary of the theorems of Zel'manov mentioned in the Introduction. This proposition is 'folklore'.

Proposition 2.1. There exist numbers $l=l(n)$ and $m=m(n)$ such that the law

$$
\left[x_{1}, x_{2}, \ldots, x_{m+1}\right]^{l}=1
$$

holds in all locally nilpotent $n$-Engel groups.

Proof. By Theorem Z3 mentioned in the Introduction, every torsion free locally nilpotent $n$-Engel group is nilpotent of bounded class, say $m=m(n)$. Let $F$ be the free $n$-Engel group on $m+1$ generators, say $x_{1}, \ldots, x_{m+1}$. Let $R=\bigcap_{i=1}^{\infty} \gamma_{i}(F)$. It is clear that $F / R$ is residually nilpotent and as every finitely generated nilpotent group is residually finite it follows that $F / R$ is residually finite. By Wilson's theorem (Theorem $\mathrm{W}$ ), $F / R$ is then nilpotent. Let $T / R$ be the torsion group of $F / R$. Now 
$F / T$ is a torsion free nilpotent $n$-Engel group and by the remark at the beginning of the proof, $F / T$ is thus nilpotent of class at most $m$. So $\left[x_{1}, \ldots, x_{m+1}\right]^{l} \in R$ for some positive integer $l=l(n)$.

Now let $G$ be any locally nilpotent $n$-Engel group and let $g_{1}, \ldots, g_{m+1} \in G$. There is a homomorphism

$$
\phi: F \longrightarrow G, \quad \phi\left(x_{i}\right)=g_{i}, \quad i=1,2, \ldots, m+1 .
$$

As $\left\langle g_{1}, \ldots, g_{m+1}\right\rangle=\phi(F)$ is nilpotent, we have that $R \leq \operatorname{Ker}(\phi)$. Hence $1=$ $\phi\left(\left[x_{1}, \ldots, x_{m+1}\right]^{l}\right)=\left[g_{1}, \ldots, g_{m+1}\right]^{l}$.

We will need some theory of powerful $p$-groups. We start by reminding the reader of some definitions. Let $G$ be a finite $p$-group. If $p$ is odd, then $G$ is said to be powerful if $[G, G] \leq G^{p}$ and if $p=2$, then $G$ is powerful if $[G, G] \leq G^{4}$. We also need the notion of powerful embedding. Let $H$ be a subgroup of $G$. If $p$ is odd, then $H$ is said to be powerfully embedded in $G$ if $[H, G] \leq H^{p}$ and if $p=2$, then we require instead that $[H, G] \leq H^{4}$.

We now list some of the properties that we will be using. Let $G$ be a powerful $p$-group. If a subgroup $H$ is powerfully embedded in $G$, then $H^{p}$ is also powerfully embedded. We also have that $\left(G^{p^{i}}\right)^{p^{j}}=G^{p^{i+j}}$. Furthermore, if $G$ is generated by $x_{1}, \ldots, x_{d}$, then $G^{p}$ is and generated by $x_{1}^{p}, \ldots, x_{d}^{p}$. It follows that if $G$ is generated by elements of order dividing $p^{m}$, then $G$ has exponent dividing $p^{m}$. We also have that the terms of the lower central series are powerfully embedded in $G$. We refer to [6] or [17] for the details.

Proposition 2.2. There exists a positive integer $s=s(n)$ such that any powerful $n$-Engel p-group is nilpotent of class at most s.

Proof. By Proposition 2.1, $\left[g_{1}, \ldots, g_{m+1}\right]^{l}=1$ for all $g_{1}, \ldots, g_{m+1} \in G$, where $m$ and $l$ are the integers given in Proposition 2.1. Suppose that $v=v(n)$ is the largest exponent of the primes that appear in the decomposition of $l$. Then $\left[g_{1}, \ldots, g_{m+1}\right]^{p^{v}}=1$. So $\gamma_{m+1}(G)$ is generated by elements of order dividing $p^{v}$. But $\gamma_{m+1}(G)$ is powerfully embedded in $G$ and therefore it follows that $\gamma_{m+1}(G)$ is powerful and has exponent dividing $p^{v}$.

On the other hand, since $\gamma_{m+1}(G)$ is powerfully embedded in $G$, we have $\left[\gamma_{m+1}(G), G\right] \leq \gamma_{m+1}(G)^{p}$ if $p$ is odd, and $\left[\gamma_{m+1}(G), G\right] \leq \gamma_{m+1}(G)^{4}$ if $p=2$. Using some basic properties of powerful groups we see inductively that

$$
\left[\gamma_{m+1}(G),{ }_{v} G\right] \leq \gamma_{m+1}(G)^{p^{v}}=1 .
$$

Hence, $G$ is nilpotent of class at most $s(n)=m+v$.

Proposition 2.3. Let $G$ be a finite n-Engel p-group.

(a) If $p$ is odd, then $G^{p^{r}}$ is powerful.

(b) If $p=2$, then $\left(G^{2^{r}}\right)^{2}$ is powerful.

Proof. Suppose that $K \leq H$ are two normal subgroups of $G$ such that $H / K$ is elementary abelian, and $x$ is an arbitrary element of $G$. Put $t=x K$ and $V=H / K$. Since $[V, n t]=1$, we have that $\left[V, p^{r} t\right]=1$ and $0=(t-1)^{p^{r}}=t^{p^{r}}-1$ in End $(V)$. Therefore $G^{p^{r}}$ centralises every elementary abelian normal section of $G$. By a lemma of A. Shalev [20], this implies that $G^{p^{r}}$ is powerful if $p$ is odd and $\left(G^{2^{r}}\right)^{2}$ is powerful if $p=2$. 
Theorem 2.4. Let $G$ be a locally finite $n$-Engel p-group.

(a) If $p$ is odd, then $G^{p^{r}}$ is nilpotent of n-bounded class.

(b) If $p=2$, then $\left(G^{2^{r}}\right)^{2}$ is nilpotent of $n$-bounded class.

Proof. First suppose that $p$ is odd. Let $g_{1}, \ldots, g_{s+1} \in G^{p^{r}}$ where $s=s(n)$ is as in Proposition 2.2. Now $g_{1}, \ldots, g_{s+1} \in H^{p^{r}}$ for some finitely generated subgroup $H$ of $G$. As $G$ is locally finite, $H$ is finite. It follows by Proposition 2.3 that $H^{p^{r}}$ is powerful. By Proposition 2.2 we can deduce that $H^{p^{r}}$ is nilpotent of class at most $s$. Hence $\left[g_{1}, \ldots, g_{s+1}\right]=1$. This shows that $G^{p^{r}}$ is nilpotent of class at most $s$. The proof for $p=2$ is similar.

Remark. Suppose that $n, p$ are such that $r=r(n, p) \geq 2$ and

$$
G=\mathbb{Z}_{p} \mathrm{wr} \bigoplus_{i=1}^{\infty} \mathbb{Z}_{p^{r-1}}
$$

Then $G$ is a metabelian $n$-Engel $p$-group of exponent $p^{r}$ such that $G^{p^{r-2}}$ is not nilpotent. Therefore, if $t$ is the least non-negative integer such that $G^{p^{t}}$ is nilpotent, for all locally finite $n$-Engel $p$-groups $G$, then we have $t \in\{r-1, r\}$ if $p$ is odd and $t \in\{r-1, r, r+1\}$ if $p=2$.

We end this section by an application which is of independent interest. A group is said to be $(d, m)$ nilpotent if every $d$-generator subgroup is nilpotent of class at most $m$. In [7, G. Endimioni posed the question of whether every $(d, 3 d-3)$ nilpotent 2-group $G$ is soluble. He also proved that this is true when $G$ is of finite exponent. As a corollary to our work we show that this is true in general. Such groups are locally finite [8], and by Proposition $2.4, G^{2^{r}}$ is nilpotent for some $r$. So the problem is reduced to the case when $G$ is of finite exponent. Hence the result.

We remark that the free group $B(\infty, 4)$ of exponent 4 with countable rank is $(d, 3 d-2)$ nilpotent for $d \geq 3$ [11, [18]. As the group $B(\infty, 4)$ is non-solvable [18, it follows that $3 d-3$ is the best upper bound in the result above provided that $d \geq 3$. Endimioni [7] asked whether this is also the case when $d=2$. As $B(\infty, 4)$ is not $(2,4)$ nilpotent this is not immediately clear. However, one can see from a power commutator presentation of the free 3-generator group of exponent 4 that $B(\infty, 4) / Z(B(\infty, 4))$ is $(2,4)$ nilpotent $[3$. So $3 d-3$ is also the best upper bound when $d=2$.

\section{4-ENGEL 3-GROUPS}

In this section we shall prove that every 4-Engel 3-group is soluble. The following arguments will involve 2-Engel groups. We first introduce a class of groups $E_{2}$. We will see later that $E_{2}$ coincides with the class of 2-Engel groups.

Definition. A group $G$ is said to be an $E_{2}$-group if $G$ is a product of a family of normal abelian subgroups $\left(H_{i}\right)_{i \in I}$ and

$$
[a, b, c][a, c, b]=1
$$

for all $a, b, c \in \bigcup_{i \in I} H_{i}$.

Remark. Suppose that $H_{i}$ is generated by $X_{i}$. Then it is easy to see that, in the definition above, it is sufficient to assume that $[a, b, c][a, c, b]=1$ for all $a, b, c \in$ $\bigcup_{i \in I} X_{i}$. We will be using this later without mention. 
Lemma 3.1. If $G$ is an $E_{2}$-group, then $G$ is nilpotent of class at most 3 .

Proof. Let $\left(H_{i}\right)_{i \in I}$ be as in the definition. Take $a, b, c \in \bigcup_{i \in I} H_{i}$. By (1) we have that $[a, b, c]=[a, c, b]^{-1}=[c, a, b]$ and thus

$$
[a, b, c]=[c, a, b]=[b, c, a] .
$$

Notice that $\bigcup_{i \in I} H_{i}$ is closed under taking commutators. Now let $a, b, c, d \in$ $\bigcup_{i \in I} H_{i}$. By using (1) and (2) we have

$$
\begin{aligned}
{[[a, b], c, d] } & =[c, d,[a, b]] \\
& =[c, d, a, b][c, d, b, a]^{-1}=[c, d, a, b]^{2}
\end{aligned}
$$

and also

$$
\begin{aligned}
{[[a, b, c], d] } & =[[c, a, b], d]=[[c, a], b, d] \\
& =[[c, a], d, b]^{-1}=[c, a, d, b]^{-1}=[c, d, a, b] .
\end{aligned}
$$

Comparing these two calculations we have $[c, d, a, b]=1$. As $G$ is generated by $\bigcup_{i \in I} H_{i}$, it follows that $G$ is nilpotent of class at most 3 .

Lemma 3.2. A group is an $E_{2}$-group if and only if it is 2-Engel.

Proof. First suppose that $G$ is 2-Engel. Let $a, b, c \in G$. We know that $G$ is nilpotent of class at most $3[16]$. Therefore $1=[a, b c, b c]=[a, b, c][a, c, b]$. In every 2-Engel group, the normal closure of an element is abelian. Thus every 2-Engel group is a product of normal abelian subgroups. Hence $G$ is an $E_{2}$-group.

Conversely, suppose that $G$ is an $E_{2}$-group. Now let $a=\prod_{i \in I} a_{i}$ and $b=\prod_{i \in I} b_{i}$ be elements of $G$, where $a_{i}, b_{i} \in H_{i}$ and for almost all $i$ we have that $a_{i}=b_{i}=1$. Clearly any element in $G$ has such a representation. By Lemma 3.1 we know that $G$ is nilpotent of class at most 3 . Expanding $[a, b, b]$ we see that it is a product of elements of the form $\left[a_{i}, b_{j}, b_{k}\right]\left[a_{i}, b_{k}, b_{j}\right]$ and thus trivial.

We want to show that every 4-Engel 3-group is soluble of bounded derived length. As $3<4 \leq 3^{2}$, it follows from Theorem 2.4 that it suffices to consider groups of exponent 9 . We will see that these groups are built from 2-Engel groups.

From now on suppose that $G$ is a 4 -Engel group of exponent dividing 9 and let

$$
X=\bigcup_{a \in G}\left\langle a^{3}\right\rangle^{G}
$$

By Lemma 10 of [22] we have that $\left\langle a^{3}\right\rangle^{G}$ is abelian of exponent dividing 3 for all $a \in$ $G$. We will next perform some calculations that will eventually give us Proposition 3.6, and this proposition will enable us to derive solubility of 4-Engel 3-groups. The following calculations are modelled on calculations done by P. J. Higgins on 4-Engel Lie rings [14.

Lemma 3.3. Let $x_{1}, x_{2}, x_{3}, u, y \in X$ and let $x=x_{1} x_{2} x_{3}$. Then

$$
\begin{aligned}
{[u, x, x, x, y][u, x, x, y, x][u, x, y, x, x][u, y, x, x, x] } & =1 \\
{[u, x, x, x, y][u, x, x, y, x]^{-1}[u, y, x, x, x]^{-1} } & =1 \\
{[u, x, y, x, x][u, x, x, x, y]^{-1} } & =1 .
\end{aligned}
$$


Proof. We remark first that the group generated by $x_{1}, x_{2}, x_{3}, u, y$ is nilpotent of class at most 5 , as the normal closure of each of these elements is abelian. We get the first identity by expanding $1=\left[u,_{4} x y\right]$. We then have

$$
\begin{aligned}
1 & =[y, 4 u x] \\
& =[y, u, x, x, x][y, x, u, x, x][y, x, x, u, x][y, x, x, x, u] \\
& =[u, y, x, x, x]^{-1}[u,[y, x], x, x]^{-1}[u,[y, x, x], x]^{-1}[u,[y, x, x, x]]^{-1} \\
& =[u, y, x, x, x]^{-1}[u, x, x, y, x]^{-1}[u, x, x, x, y] .
\end{aligned}
$$

This gives the second identity and the third follows by multiplying the first two together. Note that here we have used the fact that $\langle u\rangle^{G}$ is of exponent dividing 3.

Lemma 3.4. Let $x_{1}, x_{2}, x_{3}, x_{4}, u, y \in X$ and let $x=x_{1} x_{2} x_{3} x_{4}$. Then

$$
[u, x, x, y, x, x]=[u, x, x, x, y, x]=[u, x, y, x, x, x]=1 .
$$

Proof. For $i \in\{1,2,3,4\}$, let $\tilde{x}(i)$ be the product of the elements $x_{j}, j \in\{1,2,3,4\} \backslash$ $\{i\}$. The order of the product will not matter in the following calculations. Notice also that the group generated by $x_{1}, x_{2}, x_{3}, x_{4}, u, y$ is nilpotent of class at most 6 . By (5), we have

$$
\begin{aligned}
& {\left[u, x_{i}, \tilde{x}(i), \tilde{x}(i), \tilde{x}(i), y\right]=\left[u, x_{i}, \tilde{x}(i), y, \tilde{x}(i), \tilde{x}(i)\right],} \\
& {\left[u, \tilde{x}(i), \tilde{x}(i), \tilde{x}(i), y, x_{i}\right]=\left[u, \tilde{x}(i), y, \tilde{x}(i), \tilde{x}(i), x_{i}\right] .}
\end{aligned}
$$

Now summing over $i=1,2,3,4$, gives

$$
\begin{aligned}
& {[u, x, x, x, x, y]=[u, x, x, y, x, x],} \\
& {[u, x, x, x, y, x]=[u, x, y, x, x, x] .}
\end{aligned}
$$

As $G$ is 4 -Engel it follows from the former identity that $[u, x, x, y, x, x]=1$. We next perform a similar calculations using (3) and see that

$$
\begin{aligned}
1 & =[u, x, x, x, y, x][u, x, x, y, x, x][u, x, y, x, x, x][u, y, x, x, x, x] \\
& \stackrel{(6)}{=}[u, x, x, x, y, x]^{2} .
\end{aligned}
$$

So $[u, x, x, x, y, x]=[u, x, y, x, x, x]=1$, since $\langle u\rangle^{G}$ is of exponent 3 .

Lemma 3.5. Suppose $x_{1}, x_{2}, x_{3}, y_{1}, y_{2}, y_{3} \in X, i \in\{1,2,3\}$, and let $\tilde{y}(i)$ be the product of $\left\{y_{j} \mid j \in\{1,2,3\} \backslash\{i\}\right\}$. Let $x=x_{1} x_{2} x_{3}$. Then

$$
\begin{aligned}
& 1=[u, x, x, x, \tilde{y}(i), \tilde{y}(i)][u, x, x, \tilde{y}(i), \tilde{y}(i), x][u, x, \tilde{y}(i), x, \tilde{y}(i), x][u, \tilde{y}(i), x, x, \tilde{y}(i), x] \\
& 1=[u, x, x, \tilde{y}(i), x, \tilde{y}(i)][u, x, x, \tilde{y}(i), \tilde{y}(i), x][u, x, \tilde{y}(i), \tilde{y}(i), x, x][u, \tilde{y}(i), x, \tilde{y}(i), x, x] \\
& 1=[u, x, \tilde{y}(i), x, x, \tilde{y}(i)][u, x, \tilde{y}(i), x, \tilde{y}(i), x][u, x, \tilde{y}(i), \tilde{y}(i), x, x][u, \tilde{y}(i), \tilde{y}(i), x, x, x] \\
& 1=[u, \tilde{y}(i), \tilde{y}(i), x, x, x][u, \tilde{y}(i), x, x, x, \tilde{y}(i)][u, \tilde{y}(i), x, \tilde{y}(i), x, x][u, \tilde{y}(i), x, x, \tilde{y}(i), x] \\
& 1=[u, x, x, x, \tilde{y}(i), \tilde{y}(i)][u, \tilde{y}(i), x, x, x, \tilde{y}(i)][u, x, x, \tilde{y}(i), x, \tilde{y}(i)][u, x, \tilde{y}(i), x, x, \tilde{y}(i)] \\
& {[u, \tilde{y}(i), x, x, x, \tilde{y}(i)]=[u, \tilde{y}(i), x, \tilde{y}(i), x, x]} \\
& {[u, x, x, x, \tilde{y}(i), \tilde{y}(i)]=[u, x, \tilde{y}(i), x, x, \tilde{y}(i)] .}
\end{aligned}
$$

Proof. Let $j, k \in\{1,2,3\} \backslash\{i\}$. From the first identity of Lemma 3.4 we have

$$
\begin{aligned}
1= & {\left[u, x y_{j}, x y_{j}, y_{k}, x y_{j}, x y_{j}\right] } \\
= & {\left[u, y_{j}, x, y_{k}, x, x\right]\left[u, x, y_{j}, y_{k}, x, x\right]\left[u, x, x, y_{k}, y_{j}, x\right] } \\
& {\left[u, x, x, y_{k}, x, y_{j}\right] . }
\end{aligned}
$$


Now interchange $y_{j}$ and $y_{k}$. Adding the new identity to the above gives the second identity of the Lemma. The first and third are proved similarly using the other identities of Lemma 3.4. The fourth identity comes from (3). First replace $u$ by $\left[u, y_{j}\right]$ and $y$ by $y_{k}$. Then interchange $j$ and $k$ and add. The fifth identity follows also from (3). First replace $y$ by $y_{j}$ and take commutator with $y_{k}$ from the right, then interchange $j$ and $k$ and add as before. The last two identities follow from (5) in the same way.

For the final part of the calculations, we first need some notation. Let $x_{1}, x_{2}, x_{3}$, $y_{1}, y_{2}, y_{3}$ be in $X$. We let $x=x_{1} x_{2} x_{3}$ and $y=y_{1} y_{2} y_{3}$. We give the commutators of weight 7 names as follows:

$$
\begin{array}{ll}
a=[u, x, x, x, y, y, y], & \bar{a}=[u, y, y, y, x, x, x], \\
b=[u, y, y, x, x, x, y], & \bar{b}=[u, x, x, y, y, y, x], \\
c=[u, y, x, x, x, y, y], & \bar{c}=[u, x, y, y, y, x, x], \\
d=[u, x, x, y, x, y, y], & \bar{d}=[u, y, y, x, y, x, x], \\
e=[u, y, x, y, x, x, y], & \bar{e}=[u, x, y, x, y, y, x], \\
f=[u, x, x, y, y, x, y], & \bar{f}=[u, y, y, x, x, y, x], \\
g=[u, x, y, y, x, x, y], & \bar{g}=[u, y, x, x, y, y, x], \\
h=[u, x, y, x, x, y, y], & \bar{h}=[u, y, x, y, y, x, x], \\
k=[u, y, x, x, y, x, y], & \bar{k}=[u, x, y, y, x, y, x], \\
l=[u, x, y, x, y, x, y], & \bar{l}=[u, y, x, y, x, y, x] .
\end{array}
$$

Now take the equations of Lemma 3.5 and commute them on the right by $y_{i}$. Adding for $i=1,2,3$ gives the following 7 identities:

$$
\begin{aligned}
& a f k l=1, \quad \operatorname{def} g=1, \quad b g h l=1, \quad b c e k=1, \\
& a c d h=1, \quad c=e, \quad a=h .
\end{aligned}
$$

By symmetry, there are similar identities for $\bar{a}, \ldots, \bar{l}$. Solving these linear equations together gives

$$
\begin{array}{lll}
d=a c^{-1}, & e=c, \quad f=a b c, \quad g=a b^{-1} c^{-1}, \\
h=a, & k=b^{-1} c, \quad l=a c .
\end{array}
$$

Similarly for $\bar{a}, \ldots, \bar{l}$. Next we take the equations in Lemma 3.5 and replace $u$ by $\left[u, y_{i}\right]$. Adding for $i=1,2,3$ gives

$$
\begin{aligned}
& 1=c \bar{g} \bar{l} \bar{f}=c \bar{c}, \quad 1=k \bar{g} \bar{h} \bar{d}=b^{-1} \bar{b}^{-1} c \bar{c}, \quad 1=\bar{a} b \bar{d} \bar{f}=b \bar{b}, \quad 1=e \bar{l} \bar{h} \bar{a}=c \bar{c}, \\
& 1=c b k e, \quad b=\bar{d}=\bar{a} \bar{c}^{-1}, \quad c=e .
\end{aligned}
$$

By symmetry we also have $\bar{b}=a \bar{c}^{-1}$.

It now follows that $\bar{c}=c^{-1}, \bar{b}=b^{-1}$ and $\bar{a}=a^{-1}$. It is this last one that is going to give us the solubility of 4 -Engel 3 -groups. Let us state this as a proposition.

Proposition 3.6. If $x_{1}, x_{2}, x_{3}, y_{1}, y_{2}, y_{3}, u \in X, x=x_{1} x_{2} x_{3}$ and $y=y_{1} y_{2} y_{3}$, then

$$
[u, x, x, x, y, y, y][u, y, y, y, x, x, x]=1 .
$$

We can now prove the main result of this section.

Theorem 3.7. Every 4-Engel 3-group is soluble and the derived length is bounded by some constant.

Proof. First of all, we know that every 4-Engel 3-group is locally finite [22]. By Theorem 2.4 we have that $G^{9}$ is nilpotent for all 4-Engel 3-groups $G$ and the nilpotency class is bounded by some constant. It therefore suffices to consider 
a 4-Engel group $G$ of exponent 9. We know that the normal closure of the third power of any element is abelian of exponent 3 . Let $g, a_{1}, a_{2}, a_{3}, b_{1}, b_{2}, b_{3}$ be arbitrary elements in $G$ and let $a=a_{1}^{3} a_{2}^{3} a_{3}^{3}$ and $b=b_{1}^{3} b_{2}^{3} b_{3}^{3}$. By Proposition 3.6 we have

$$
\begin{aligned}
1 & =\left[g^{3}, a, a, a, b, b, b\right]\left[g^{3}, b, b, b, a, a, a\right] \\
& =\left[g^{3}, a^{3}, b^{3}\right]\left[g^{3}, b^{3}, a^{3}\right] .
\end{aligned}
$$

For each $a_{1}, a_{2}, a_{3} \in G$ we let $H\left(a_{1}, a_{2}, a_{3}\right)=\left\langle\left(a_{1}^{3} a_{2}^{3} a_{3}^{3}\right)^{3}\right\rangle^{G}$ and then

$$
H=\prod_{\left(a_{1}, a_{2}, a_{3}\right) \in G \times G \times G} H\left(a_{1}, a_{2}, a_{3}\right) .
$$

In other words $H$ is the verbal subgroup generated by the word $\left(x_{1}^{3} x_{2}^{3} x_{3}^{3}\right)^{3}$. We have just seen that Proposition 3.6 implies that $H$ is an $E_{2}$-group and thus 2-Engel. Now consider $G / H$. For all $g_{1}, g_{2}, g_{3} \in G$ we have

$$
\begin{aligned}
& \left(g_{1}^{3} g_{2}^{3} g_{3}^{3}\right)^{3}=\left(g_{1}^{3} g_{2}^{3}\right)^{3}\left(g_{3}^{3}\right)^{\left(g_{1}^{3} g_{2}^{3}\right)^{2}+\left(g_{1}^{3} g_{2}^{3}\right)+1} \\
& =\left[g_{3}^{3}, g_{1}^{3} g_{2}^{3}, g_{1}^{3} g_{2}^{3}\right] \\
& =\left[g_{3}^{3}, g_{1}^{3}, g_{2}^{3}\right]\left[g_{3}^{3}, g_{2}^{3}, g_{1}^{3}\right] .
\end{aligned}
$$

So $G^{3} / H$ satisfies the identity

$$
1=\left[g^{3}, a^{3}, b^{3}\right]\left[g^{3}, b^{3}, a^{3}\right] .
$$

This shows that $G^{3} / H$ is also 2-Engel. Finally $G / G^{3}$ is of exponent 3 and thus 2-Engel [16. Thus $G / G^{3}, G^{3} / H$ and $H$ are 2-Engel and thus metabelian and it follows that $G$ is soluble of derived length at most 6 .

Remark. The referee has pointed out to us that in 4-Engel groups of exponent 9, a stronger result holds than the one we obtained. One can show that $G^{3}$ is 2-Engel with a computer using the $p$-quotient algorithm. It follows that the derived length of these groups is at most 4 .

As a corollary of Theorem 3.7 we have

Theorem 3.8. Let $G$ be a locally nilpotent 4-Engel group without elements of order 2 or 5 . Then $G$ is soluble and the derived length is bounded by some constant.

Proof. Let $\mathcal{P}$ be the set of all primes and let $\Pi=\mathcal{P} \backslash\{2,5\}$. We say that a finite group $H$ is a $\Pi$-group if the order of any element in $H$ has all its prime divisors from $\Pi$.

Let $H$ be a finite 4-Engel $p$-group for some $p \in \Pi$. If $p \neq 3$, we know that $H$ is nilpotent of class at most 7 [22] and if $p=3$, we have seen that $H$ is soluble of some derived length bounded by some constant. So in any case, we have that $H$ is soluble and the derived length is bounded by some constant $m$. By a theorem of Zorn (see [19, 12.3.4]), all finite 4-Engel groups are nilpotent and so it follows that every finite 4-Engel $\Pi$-group is soluble of derived length at most $m$. We finish the proof by showing that $G$ is also soluble of derived length at most $m$.

Clearly we can assume that $G$ is generated by $2^{m}$ elements and thus that $G$ is a finitely generated nilpotent group. Let $\tau(G)$ be the torsion subgroup of $G$. It now suffices to show that $G$ is residually a finite $\Pi$-group.

Let $1 \neq x \in G$. We need to find a normal subgroup $M$ such that $G / M$ is a finite $\Pi$-group and $x \notin M$. We consider two cases. First suppose that $x$ has infinite order. Then $G / \tau(G)$ is a finitely generated torsion-free nilpotent group and thus 
residually a finite $p$-group for any prime $p$ (see [19, 5.2.22]). In particular we can choose $p \in \Pi$ and a normal subgroup $M$ of $G$ with $G / M$ a finite $p$-group and $x \notin M$.

We are left with the case when $x$ has finite order. As $G$ is a finitely generated nilpotent group, it is residually finite (see [19, 5.4.17]). Therefore there exists a normal subgroup $M$ of $G$ and some prime $p$ such that $G / M$ is a finite $p$-group and $x \notin M$. As the order of $x M$ in $G / M$ divides the order of $x$ we must have $p \in \Pi$.

\section{REFERENCES}

1. S. Bachmuth, Exceptional primes in varieties, in "Conference on Group Theory (Univ. Wisconsin-Parkside 1972)", Lecture Notes in Math. 319, Springer-Verlag (1973), 19-25. MR 51:8253

2. S. Bachmuth and H. Y. Mochizuki, Third Engel groups and the Macdonald-Neumann conjecture, Bull. Austral. Math. Soc. 5 (1971), 379-386. MR 46:1917

3. A. J. Bayes, J. Kautsky and J. W. Wamsley, Computation in nilpotent groups (application), in "Proceedings of the Second International Conference on the Theory of Groups (Australian Nat. Univ. Canberra 1973)", Lecture Notes in Math., Vol. 372, Springer-Verlag (1974), 82-89. MR 50:7299

4. R. G. Burns, O. Macedońska and Y. Medvedev, Groups satisfying semigroup laws, and nilpotent-by-Burnside varieties, J. Algebra 195 (1997), 510-525. MR 98h:20039

5. R. G. Burns and Y. Medvedev, A note on Engel groups and local nilpotence, J. Austral. Math. Soc. (Series A) 64 (1998), 92-100. MR 98k:20063

6. J. D. Dixon, M. P. F. du Sautoy, A. Mann and D. Segal, Analytic pro-p Groups. London Mathematical Society Lecture Note Series, 157, Cambridge University Press (1991). MR 2000m:20039

7. G. Endimioni, Sur la résolubilité des 2-groupes, C. R. Acad. Sci. Paris (Série I), 316 (1993), 1253-1255. MR 94g:20029

8. G. Endimioni, Groups in which every $d$-generator subgroup is nilpotent of bounded class, Quart. J. Math. Oxford (2) 46 (1995), 433-435. MR 97e:20037

9. M. I. Golovanov, On the degree of nilpotency of 4-Engel Lie rings, Algebra $i$ Logika 25 (1986), 508-532. MR 89b:17018

10. N. D. Gupta, Third-Engel 2-groups are soluble, Can. Math. Bull. 15 (1972), 523-524. MR 47:351

11. N. D. Gupta and M. F. Newman, The nilpotency class of finitely generated groups of exponent 4, Lecture Notes in Mathematics 372, Springer-Verlag (1974), 330-332. MR 50:4752

12. N. D. Gupta and M. F. Newman, Third Engel groups, Bull. Austral. Math. Soc. 40 (1989), 215-230. MR 91b:20036

13. H. Heineken, Engelsche Elemente der Länge drei, Illionis J. Math. 5 (1961), 681-707. MR 24:A1319

14. P. J. Higgins, Lie rings satisfying the Engel condition, Proc. Camb. Phil. Soc. 50 (1954), 8-15. MR 15:596b

15. L. C. Kappe and W. P. Kappe, On three-Engel groups, Bull. Austral. Math. Soc. 7 (1972), 391-405. MR 47:3550

16. F. W. Levi, Groups in which the commutator operation satisfies certain algebraic conditions, J. Indian Math. Soc. 6 (1942), 87-97. MR 4:133i

17. A. Lubotzky and A. Mann, Powerful p-groups. I. Finite groups, J. Algebra 105 (1987), 484505. MR 88f:20045

18. Ju. P. Razmyslov, The Hall-Higman problem, Izv. Akad. Nauk SSSR Ser. Math 42 (1978), 833-847. MR 80d:20040

19. D. J. S. Robinson, A course in the theory of groups, (Springer-Verlag, New York, 1982). MR 84k:20001

20. A. Shalev, Characterization of $p$-adic analytic groups in terms of wreath products, J. Algebra 145 (1992), 204-208. MR 92i:20029

21. G. Traustason, Engel Lie-algebras, Quart. J. Math. Oxford (2) 44 (1993), 355-384. MR 95a: 17015

22. G. Traustason, On 4-Engel groups, J. Algebra 178 (1995), 414-429. MR 96i:20049

23. Unsolved problems in group theory. The Kourovka notebook (13th edition). Edited by V.D. Mazurov and E.I. Khukhro (Novosibirsk, 1995). MR 97d:20001 
24. M. Vaughan-Lee, Engel-4 groups of exponent 5, Proc. London Math. Soc. (3) 74 (1997), 306-334. MR 98a:20036

25. J. S. Wilson, Two-generator conditions for residually finite groups, Bull. London Math. Soc. 23 (1991), 239-248. MR 92k:20068

26. E. I. Zel'manov, Engel Lie-algebras, Dokl. Akad. Nauk SSSR 292 (1987), 265-268. MR 88d:17009

27. E. I. Zel'manov, Some problems in the theory of groups and Lie algebras, Math. Sb. 180 (1989), 159-167. MR 90f:17024

28. E. I. Zel'manov, The solution of the restricted Burnside problem for groups of odd exponent, Math. USSR Izvestia 36 (1991), 41-60. MR 91i:20037

29. E. I. Zel'manov, The solution of the restricted Burnside problem for 2-groups, Mat. Sb. 182 (1991), 568-592. MR 93a:20063

Department of Mathematics, University of Isfahan, Isfahan 81744, Iran

E-mail address: alireza_abdollahi@yahoo.com

C.M.I.-Université de Provence, UMR-CNRS 6632, 39, Rue F. Joliot-Curie, 13453 MarSeille Cedex 13, France

E-mail address: gunnar@gyptis.univ-mrs.fr

Current address: Department of Mathematics, Lund Institute of Technology, P.O. Box 118, S-22100 Lund, Sweden

E-mail address: gt@maths.lth.se 